\title{
O Acesso Aberto no Turismo: Resultados preliminares
}

\author{
Cristina Marques Gomes \\ Universidade Federal de Santa Maria \\ E-mail: cristina@usp.br
}

\begin{abstract}
Resumo
Esta pesquisa (financiada pelo Conselho Nacional de Desenvolvimento Científico e Tecnológico - CNPq - Chamada Universal - MCTI/CNPq No 14/2013 - Brasil) tendo como objeto o open acess, apresenta os seguintes objetivos específicos: 1. Identificar e analisar o "quê" os pesquisadores e editores de Turismo no Brasil conhecem sobre o acesso aberto. 2. Averiguar as "motivações" e "barreiras" dos pesquisadores, em Turismo no Brasil, em relação à publicação em acesso aberto. Em termos metodológicos, um primeiro questioná-

Rede de Pesquisadores Acadêmicos em Turismo (REPETUR-Brasil) tendo como fonte/referência o trabalho português "Os Investigadores em Portugal e a sua Relação com o Acesso Aberto à Produção Científica". Outras etapas da pesquisa ainda estão em andamento e/ou por serem executadas. Como contributos finais, trará maior visibilidade sobre o tema na área de Turismo (os pesquisadores que não conhecem o movimento poderão integrar o mesmo) e proporcionará dados confiáveis para o futuro planejamento e prognóstico das publicações científicas brasileiras.
\end{abstract} rio foi aplicado em fevereiro de 2015 à

Palavras-chave: Acesso aberto; Turismo; Brasil e Portugal.

\begin{abstract}
This research (funded by the National Scientific and Technological Development Council - CNPq - Call Universal MCTI / CNPq No. 14/2013 - Brazil) having as object the open acess, has the following specific objectives: 1 . To identify and analyze "what"Tourism researchers

and editors in Brazil know about open access. 2. Identify the "motives"and "barriers" of researchers in Tourism in Brazil, concerning the publication in open access. In terms of methodology, a first questionnaire was administered in February 2015 to the researchers of the Aca-
\end{abstract}


demic Network on Tourism (REPETURBrazil) having as source/reference the Portuguese work "The Researchers in Portugal and its relationship with the Open Access to Scientific Production". Other stages of the research still in progress. As final contributions will bring greater visibility on the topic in the Tourism area (the researchers who did not know the move will integrate the same) and provide reliable data for future planning and prognosis of Brazilian scientific publications.

Keywords: Open access; Tourism; Brazil and Portugal.

\title{
Introdução
}

\begin{abstract}
discussão em torno do acesso aberto dominou praticamente todos os A campos científicos e diversos atores sociais da comunicação científica (CC) - como as bibliotecas, editoras e agências de financiamento - em âmbito internacional. Nos últimos anos, por conseguinte, todo esse contexto externo começou a reverberar no Brasil através do aumento do número de eventos acadêmicos, publicações, políticas, etc. Apesar dessa maximização de debates e ações em torno do open access, de maneira geral, não encontramos quase nenhum trabalho que conjugue este com a área de Turismo. Ao mesmo tempo, o Turismo, no Brasil, é uma disciplina que, cada vez mais, está expandindo o número de periódicos em acesso aberto - o estudo de Miranda ${ }^{1}$ de 2012, indica a existência de 44 revistas e, dessas, 19 são ativas. Ou seja, na prática o acesso aberto é implementado pela ótica dos periódicos - inclusive todos seguem o modelo de negócios do "acesso aberto total" - sem, no entanto, nenhum dado, exame, debate por parte dos pesquisadores e editores em Turismo no Brasil. Tudo são suposições e o futuro da publicação científica, na área, em termos de acesso, difusão e mesmo preservação, depende, sobremaneira, de evidências mais concretas/racionais sobre esse tema, nesse sentido, indagamos:

- Mediante a importância do OA na contemporaneidade, como se sustenta - em termos de informações teóricas sobre o acesso aberto - o conhecimento dos editores e pesquisadores em Turismo no Brasil?
\end{abstract}

1. MIRANDA, E. C. P. (2012). Comunicação Científica em Turismo e o Movimento do Acesso Aberto. In: Congresso Brasileiro de Turismólogos, Belo Horizonte. 
- E, de forma complementar, pela outra via, quais são as crenças e atitudes, traduzidas em "motivações" e "barreiras", dos pesquisadores, desta área, em relação à publicação em acesso aberto?

A partir, portanto, dessas questões, o presente artigo é dividido nos seguintes itens: "objetivos", "considerações metodológicas", "dados descritivos dos resultados preliminares: questionário com os pesquisadores da área de Turismo no Brasil" além das "considerações finais" sobre o tema.

\section{Objetivos}

Objetivo geral: Traçar o perfil do conhecimento teórico absorvido pelos "stakeholders" (pesquisadores e editores) sobre a questão do acesso aberto em Turismo no Brasil e, de forma complementar, entender as "motivações" e "barreiras", dos pesquisadores brasileiros em Turismo, em relação à publicação em acesso aberto.

Objetivos específicos/metas: 1. Identificar e analisar o "quê" os pesquisadores e editores de Turismo no Brasil conhecem sobre o acesso aberto. 2. Averiguar as "motivações" e "barreiras" dos pesquisadores, em Turismo no Brasil, em relação à publicação em acesso aberto.

\section{Considerações metodológicas}

Boa parte da metodologia inicialmente planejada advinha da experiência do Projeto "Study of Open Access Publishing" (Soap) ${ }^{2}$ associado ao "Seventh Framework Programme" (FP7), no entanto, a pesquisa no contexto brasileiro foi limitada em função da falta de capital financeiro, visto que, do montante de 29.977,44 (vinte e nove mil, novecentos e setenta e sete reais e quarenta e quatro centavos) obtivemos o financiamento de 8.700 (oito mil e setecentos reais) - sendo somente 2.700 (dois mil e setecentos reais) de custeio inviabilizando, consequentemente, a viagem para a Suíça. Por outro lado, é de grande valia a organização de estudos/pesquisas que englobem, além de objetos semelhantes, a questão da mesma língua - nesse caso, a portuguesa. Por conseguinte, o projeto, em uma de suas fases, foi direcionado para uma análise comparativa com os resultados obtidos pelo trabalho português "Os Investigadores em Portugal e a sua Relação com o Acesso Aberto à Produção

2. Disponível em: http://project-soap.eu. 
Científica". Um primeiro questionário foi aplicado, via o site survey mon$k e y^{3}$, em fevereiro de 2015 à Rede de Pesquisadores Acadêmicos em Turismo (REPETUR-Brasil) e as questões foram divididas em duas partes - com 10 perguntas cada - sendo que a primeira obteve, até o presente momento, 46 e a segunda, 35 respostas. Os pormenores desses dados serão expostos no item seguinte do presente artigo.

Agregados a esses aspectos, o projeto engloba, ainda:

a) pesquisa bibliográfica: com o propósito de identificarmos outros estudos específicos sobre acesso aberto que, por ventura, tenham sido direcionados a disciplinas específicas (Física, Psicologia e/ou qualquer outra), em âmbito internacional, nos últimos 3 anos. Será dividida em quatro etapas, são elas: "características macro do universo"; "delineamento dos domínios"; "conjunto nuclear de investigações" e "matrizes temáticas e de conceitos" - nos moldes proferidos por Webster e Watson (2002) em "Analyzing the past to prepare for the future: writing a literature review";

b) diálogo com pesquisadores portugueses - e aqui cabe salientar que Portugal é um país de referência e pioneiro no desenvolvimento do open access em escala internacional - incluindo: pesquisa qualitativa; leitura do material disponível no site do "Projetos Open Access da Universidade do Minho" ${ }^{4}$; elaboração metodológica dos dados a serem coletados na visita de campo; viagem - diálogo com um ou mais membro da equipe do "Projetos Open Access da Universidade do Minho" - realização de entrevistas não-estruturadas; tratamento e organização do material; análise e interpretação;

c) Pesquisa com editores da área de Turismo no Brasil: elaboração do questionário; realização do pré-teste; aplicação do questionário; tratamento dos dados; análise e interpretação.

3. Disponível em: https://pt.surveymonkey.com.

4. Disponível em: http://openaccess.sdum.uminho.pt. 


\section{Dados descritivos dos resultados preliminares: questionário com os pes- quisadores da área de turismo do Brasil}

As 4 primeiras questões estavam diretamente relacionadas ao perfil do entrevistado, ou seja, a idade, sexo, escolaridade e profissão e foram assim respondidas:

a) a maioria $(55,56 \%)$ estava na faixa de $31-40$ anos (25 respondentes); em seguida figuravam os respondentes (10) de 41-50 anos; depois, os até 30 anos (4 respondentes; 8,89\%), e, por fim, numericamente iguais (3 respondentes; 6,67\%) surgem as categorias "de 51-60 anos" e "mais de 60 anos";

b) sobre sexo: $51,11 \%$ era feminino e $48,89 \%$ masculino - praticamente iguais, 23 e 22 respondentes, respectivamente;

c) a escolaridade segue a seguinte sequência: doutor $(31,11 \%)$, mestre (26,67\%), doutorando $(24,44 \%)$, pós-doutorado $(15,56 \%)$ e graduação $(0,0 \%)$. Somando "doutor" e "doutorando" temos $55,55 \%$ do total de respondentes.

d) quase todos os respondentes eram docentes da área (36); seguidos de turismólogos (2) e geógrafos (2); e, por fim, com uma resposta cada, estavam as categorias "aposentado"; "guia de turismo e agente de viagem" e "analista de políticas sociais" - essa questão era aberta.

A quinta pergunta era: “concorda com a seguinte afirmação 'não existe qualquer problema com o acesso aberto à informação científica no meu país'"? E, nesse quesito, $67,39 \%$ responderam que discordam; $17,39 \%$ discordam plenamente; $13,04 \%$ concordam; $2,17 \%$ concordam plenamente e nenhum respondente assinalou a alternativa "sem opinião".

Quanto a "qual é a sua opinião em relação às seguintes potenciais barreiras de acesso às publicações científicas?" temos o seguinte quadro: 
Tabela 1. resposta questão 6 - primeira parte do questionário

\begin{tabular}{|c|c|c|c|c|c|c|c|}
\hline$=$ & $\begin{array}{l}\text { Muito } \\
\text { importante }\end{array}$ & Importante - & $\begin{array}{l}\text { Pouco } \\
\text { importante }\end{array}$ & $\begin{array}{l}\text { Nada } \\
\text { importante }\end{array}$ & $\begin{array}{l}\text { Sem } \\
\text { opiniäo }\end{array}$ & Total - & $\begin{array}{l}\text { Média } \\
\text { ponderada }\end{array}$ \\
\hline $\begin{array}{l}\text { Insuficientes } \\
\text { politicas, } \\
\text { estratégias e } \\
\text { estruturas de } \\
\text { acesso às } \\
\text { publicaçôes } \\
\text { cientificas }\end{array}$ & $\begin{array}{r}34,78 \% \\
16\end{array}$ & $\begin{array}{r}58,70 \% \\
27\end{array}$ & $\begin{array}{r}4,35 \% \\
2\end{array}$ & $\begin{array}{r}0,00 \% \\
0\end{array}$ & $\begin{array}{r}2,17 \% \\
1\end{array}$ & 46 & 1,76 \\
\hline $\begin{array}{l}\text { Elevados preços } \\
\text { dos } \\
\text { artigos/assinaturas } \\
\text { das revistas } \\
\text { cientificas }\end{array}$ & $\begin{array}{r}50,00 \% \\
23\end{array}$ & $\begin{array}{r}36,96 \% \\
17\end{array}$ & $\begin{array}{r}10,87 \% \\
5\end{array}$ & $\begin{array}{r}2,17 \% \\
1\end{array}$ & $\begin{array}{r}0,00 \% \\
0\end{array}$ & 46 & 1,65 \\
\hline $\begin{array}{l}\text { Financiamento das } \\
\text { bibliotecas } \\
\text { reduzido ou } \\
\text { limitado }\end{array}$ & $\begin{array}{r}45,65 \% \\
21\end{array}$ & $\begin{array}{r}45,65 \% \\
21\end{array}$ & $\begin{array}{r}2,17 \% \\
1\end{array}$ & $\begin{array}{r}4,35 \% \\
2\end{array}$ & $\begin{array}{r}2,17 \% \\
1\end{array}$ & 46 & 1,72 \\
\hline $\begin{array}{l}\text { Falta de interesse } \\
\text { e conscientização } \\
\text { da comunidade } \\
\text { cientifica } \\
\text { relativamente ao } \\
\text { acesso aberto }\end{array}$ & $\begin{array}{r}51,11 \% \\
23\end{array}$ & $\begin{array}{r}28,89 \% \\
13\end{array}$ & $\begin{array}{r}17,78 \% \\
8\end{array}$ & $\begin{array}{r}2,22 \% \\
1\end{array}$ & $\begin{array}{r}0,00 \% \\
0\end{array}$ & 45 & 1,71 \\
\hline $\begin{array}{l}\text { Falta de incentivos } \\
\text { para encorajar } \\
\text { práticas que } \\
\text { promovam o } \\
\text { acesso à produção } \\
\text { cientifica }\end{array}$ & $\begin{array}{r}58,70 \% \\
27\end{array}$ & $\begin{array}{r}32,61 \% \\
15\end{array}$ & $\begin{array}{r}6,52 \% \\
3\end{array}$ & $\begin{array}{r}0,00 \% \\
0\end{array}$ & $\begin{array}{r}2,17 \% \\
1\end{array}$ & 46 & 1,54 \\
\hline
\end{tabular}

"Elevados preços dos artigos/assinaturas das revistas científicas", "Falta de incentivos para encorajar práticas que promovam o acesso à produção científica" e "falta de interesse e conscientização da comunidade científica relativamente ao acesso aberto" foram apontadas como "muito importantes"; "financiamento das bibliotecas reduzido ou limitado" adquiriu de forma equivalente a mesma porcentagem nas categorias "muito importante" e "importante". E "Insuficientes políticas, estratégias e estruturas de acesso às publicações científicas" foi classificada como "importante".

O conceito de acesso aberto foi explorado em duas questões: a primeira era "conhece e compreende o conceito de acesso aberto (Open Access) à produção científica?" e a segunda "se sim, descreva". 37 responderam "sim", ou seja, $80,43 \%$ e 9 "não", correspondente a 19,57\%. As respostas descritivas englobavam: de um lado o acesso aberto de forma genérica (18 respostas - como, por exemplo, "acesso livre, transparente e gratuito"), e, de outro, o acesso aberto em oposição ao pagamento (13 respostas - como, por exemplo, "acesso aberto, sem taxas, assinaturas, etc") e, por outra via, algumas respostas (3) apresentavam um caráter mais "explicativo" - incluindo a iniciativa de um "movimento", as "pesquisas oriundas de projetos financiados por 
programas públicos" e "produzidas por instituições públicas"; e, por fim, três respostas classificadas como "contraditórias" - associando o acesso aberto com a não "avaliação prévia pelo editor e posteriormente pelos pares", outra que afirmava que "o crivo financeiro para a publicação retira a isenção das bancas julgadoras" e que "é uma plataforma online de parceria entre IES e periódicos".

A nona questão da primeira parte do questionário era "qual a sua opinião em relação às seguintes afirmações" - estas expostas no quadro abaixo:

Tabela 2. resposta questão 9 - primeira parte do questionário

\begin{tabular}{|c|c|c|c|c|c|c|c|}
\hline v & $\begin{array}{l}\text { Concordo } \\
\text { plenamente }\end{array}$ & Concordo - & $\begin{array}{l}\text { Sem } \\
\text { opinião }\end{array}$ & Discordo - & $\begin{array}{l}\text { Discordo } \\
\text { plenamente }\end{array}$ & Total - & $\begin{array}{l}\text { Média } \\
\text { ponderada }\end{array}$ \\
\hline $\begin{array}{l}\text { O acesso } \\
\text { aberto irá } \\
\text { alterara a } \\
\text { comunicação } \\
\text { científica na } \\
\text { minha área } \\
\text { de } \\
\text { investigação } \\
\text { nos próximos } \\
\text { anos }\end{array}$ & $\begin{array}{r}34,78 \% \\
16\end{array}$ & $\begin{array}{r}34,78 \% \\
16\end{array}$ & $\begin{array}{r}19,57 \% \\
9\end{array}$ & $\begin{array}{r}6,52 \% \\
3\end{array}$ & $\begin{array}{r}4,35 \% \\
2\end{array}$ & 46 & 2,11 \\
\hline $\begin{array}{l}\text { O acesso } \\
\text { aberto } \\
\text { aumenta o } \\
\text { número de } \\
\text { citaçōes dos } \\
\text { meus } \\
\text { trabalhos de } \\
\text { investigação }\end{array}$ & $\begin{array}{r}47,83 \% \\
22\end{array}$ & $\begin{array}{r}43,48 \% \\
20\end{array}$ & $\begin{array}{r}4,35 \% \\
2\end{array}$ & $\begin{array}{r}4,35 \% \\
2\end{array}$ & $\begin{array}{r}0,00 \% \\
0\end{array}$ & 46 & 1,65 \\
\hline $\begin{array}{l}\text { O acesso } \\
\text { aberto } \\
\text { aumenta o } \\
\text { acesso a } \\
\text { disseminação } \\
\text { das } \\
\text { publicações } \\
\text { científicas }\end{array}$ & $\begin{array}{r}69,57 \% \\
32\end{array}$ & $\begin{array}{r}30,43 \% \\
14\end{array}$ & $\begin{array}{r}0,00 \% \\
0\end{array}$ & $\begin{array}{r}0,00 \% \\
0\end{array}$ & $\begin{array}{r}0,00 \% \\
0\end{array}$ & 46 & 1,30 \\
\hline $\begin{array}{l}\text { O acesso } \\
\text { aberto à } \\
\text { produção } \\
\text { cientifica } \\
\text { pode } \\
\text { coexistir com } \\
\text { o tradicional } \\
\text { modelo de } \\
\text { publicação } \\
\text { cientifica }\end{array}$ & $\begin{array}{r}28,26 \% \\
13\end{array}$ & $\begin{array}{r}43,48 \% \\
20\end{array}$ & $\begin{array}{r}10,87 \% \\
5\end{array}$ & $\begin{array}{r}15,22 \% \\
7\end{array}$ & $\begin{array}{r}2,17 \% \\
1\end{array}$ & 46 & 2,20 \\
\hline
\end{tabular}

Já a décima pergunta era "qual a sua opinião sobre o princípio da disponibilização em acesso aberto das publicações científicas resultantes de projetos financiados por programas públicos?" com 39 respondentes, ou seja, 84,78\% afirmando que "concordo plenamente", 7 respondentes $(15,22 \%)$ com o "concordo" e $0 \%$ "sem opinião", "discordo" e "discordo plenamente". 
Para a segunda parte do questionário o respondente tinha que "clicar" num outro link e, dos 46 ativos, somente 35 migraram de uma parte para a outra revelando, pois, em certo sentido, o "desinteresse" da comunidade acadêmica de Turismo no Brasil pela temática do acesso aberto. Os dados descritivos são assim apresentados:

1. Como reagiria perante um mandato de acesso aberto à produção científica implementado por órgãos governamentais do seu país? (Capes, $\mathrm{CNPq}$, etc - no caso brasileiro)

Tabela 3. resposta questão 1 - segunda parte do questionário

\begin{tabular}{|l|lc}
\hline Opções de resposta & Respostas \\
\hline Cumpriria integralmente com facilidade & $82,86 \%$ & 29 \\
\hline Cumpriria integralmente com desagrado & $5,71 \%$ & 2 \\
\hline Cumpriria parcialmente & $11,43 \%$ & 4 \\
\hline Não cumpriria & $0,00 \%$ & 0 \\
\hline Total & & 35 \\
\hline
\end{tabular}

2. Na sua opinião, qual a forma mais fácil de satisfazer os requisitos de uma política obrigatória de acesso aberto à produção científica?

Tabela 4. resposta questão 2 - segunda parte do questionário



3. Na sua opinião, se o editor definir um período de tempo durante o qual sua publicação não pode estar em acesso aberto, devem ser considerados: 
Tabela 5. resposta questão 3 - segunda parte do questionário

\begin{tabular}{l|lc}
\hline Opções de resposta & Respostas \\
\hline-6 meses & $25,71 \%$ & 9 \\
\hline 9 meses & $0,00 \%$ & 0 \\
\hline$\quad 12$ meses & $11,43 \%$ & 4 \\
\hline$\quad 18$ meses & $0,00 \%$ & 0 \\
\hline$\quad$ Sem período de embargo, imediatamente disponivel & $54,29 \%$ & 19 \\
\hline$\quad$ Não sei / sem opinião & $8,57 \%$ & 3 \\
\hline Total & \multicolumn{2}{|c}{3} \\
\hline
\end{tabular}

4. O acesso aberto pode ser relevante não apenas para as publicações mas também para os dados científicos. Qual a sua opinião sobre o princípio da disponibilização em acesso aberto dos dados científicos resultantes de projetos financiados por programas públicos?

Figura 1. resposta questão 4 - segunda parte do questionário

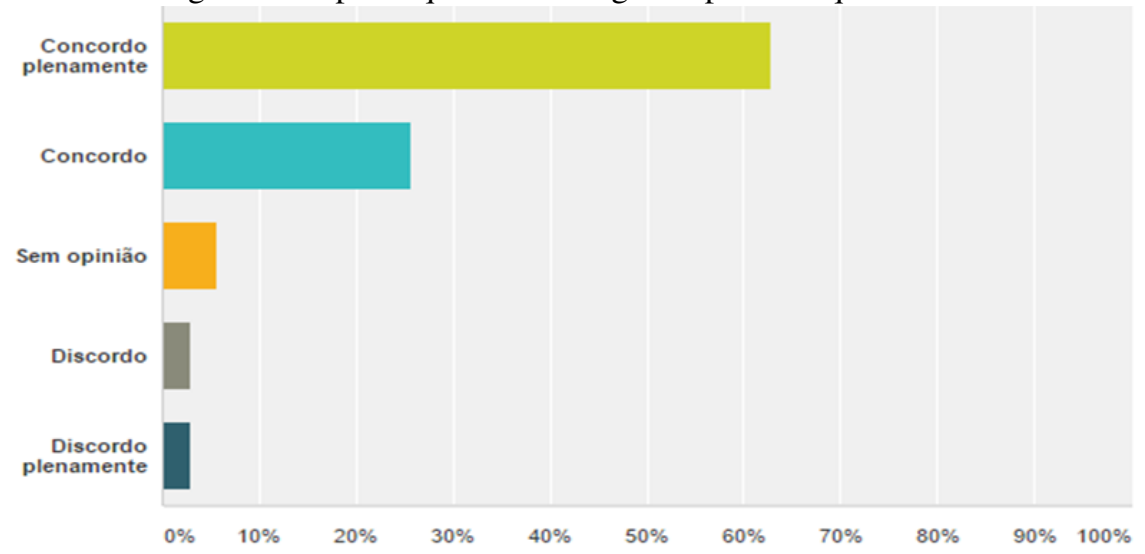

5. Indique o número de publicações que melhor define a sua produção científica anual: 
Figura 2. resposta questão 1 - segunda parte do questionário

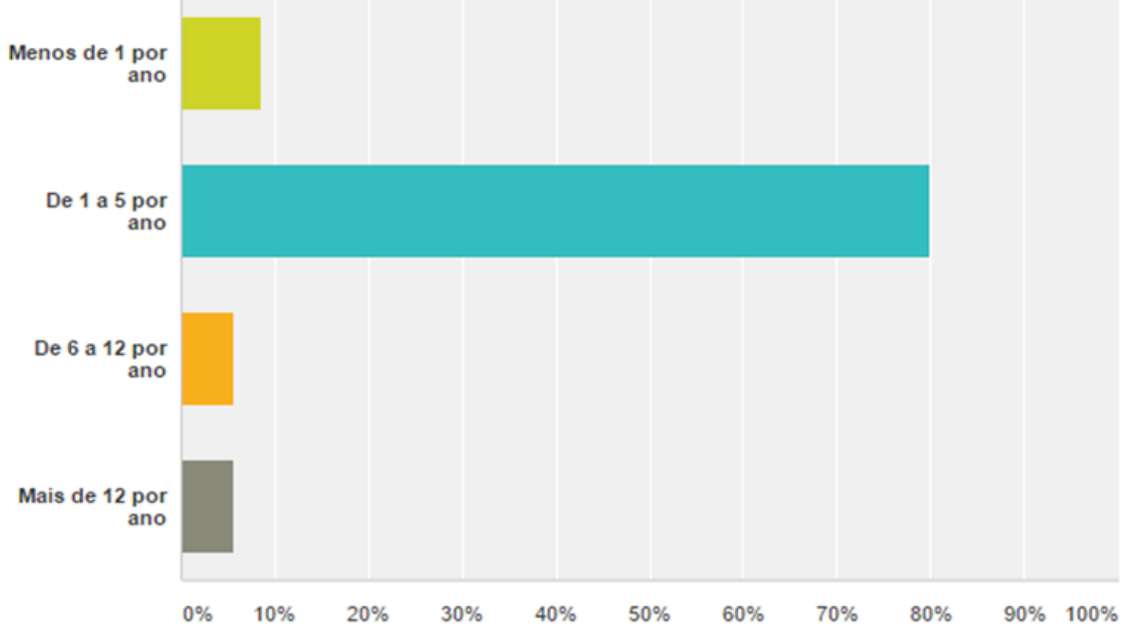

6. Já disponibilizou alguma das suas publicações em acesso aberto?

Tabela 6. resposta questão 6 - segunda parte do questionário

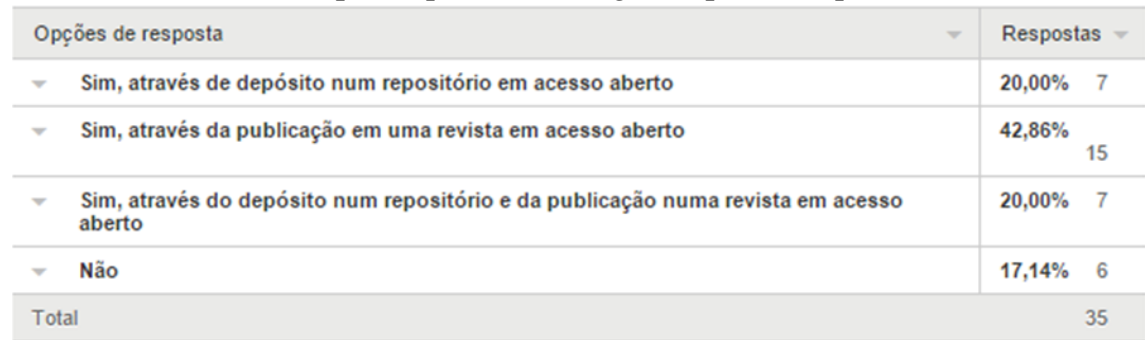

7. Indique o número aproximado dos artigos em acesso aberto: 
Tabela 7. resposta questão 7 - segunda parte do questionário

\begin{tabular}{|c|c|c|c|}
\hline Opções de resposta & $=$ & Respostas & $\checkmark$ \\
\hline Depositados em repositórios institucionais & Respostas & $62,50 \%$ & 15 \\
\hline Depositados em repositórios temáticos & Respostas & $54,17 \%$ & 13 \\
\hline Publicados em revistas cientificas em acesso aberto & Respostas & $91,67 \%$ & 22 \\
\hline
\end{tabular}

8. Na sua opinião, quais são os TRÊS principais motivos para proceder ao depósito dos seus artigos científicos em repositórios institucionais / temáticos (Indique no máximo 3 motivos)

Tabela 8. resposta questão 8 - segunda parte do questionário

\begin{tabular}{|c|c|c|c|}
\hline Opções de resposta & $\checkmark$ & Respostas & $\checkmark$ \\
\hline Aumenta a visibilidade e o impacto (citaçöes) da pesquisa & & $70,59 \%$ & 24 \\
\hline Aumenta a rapidez de disseminação dos resultados da pesquisa & & $67,65 \%$ & 23 \\
\hline É obrigatório na minha instituição & & $17,65 \%$ & 6 \\
\hline Posso delegar essa tarefa em outra pessoa & & $2,94 \%$ & 1 \\
\hline Existe suficiente suporte técnico na minha instituição & & $0,00 \%$ & 0 \\
\hline A editora autoriza o depósito em repositórios institucionais & & $0,00 \%$ & 0 \\
\hline O processo de depósito é rápido e simples & & $17,65 \%$ & 6 \\
\hline É um requisito do financiador da minha pesquisa & & $5,88 \%$ & 2 \\
\hline É uma forma de preservar a minha produção cientifica & & $50,00 \%$ & 17 \\
\hline Publico em revistas de acesso aberto & & $38,24 \%$ & 13 \\
\hline
\end{tabular}

9. Na sua opinião, quais são os TRÊS principais motivos para não depositar os seus artigos científicos num repositório institucional / temático (indique no máximo 3 motivos): 
Tabela 9. resposta questão 9 - segunda parte do questionário



A décima questão da segunda parte era "aberta" para "comentários gerais" e obteve 7 respostas completamente distintas como, por exemplo, "interessante pesquisa e interessante discussão. Falta conhecimento sobre a existência de publicações de acesso aberto no Brasil e no exterior e qual o reconhecimento que será dado pelo meio científico e pelo CAPES/CNPQ para este tipo de publicação. E como são classificadas estas publicações quanto ao Qualis?" e "acredito que o conceito de metadados no acesso aberto é algo que deve ser fortemente incentivado".

\section{Considerações finais}

Podemos afirmar que os nossos principais contributos, são: de um lado a composição do perfil do conhecimento teórico sobre o acesso aberto, por parte dos pesquisadores e editores de Turismo no Brasil que, pelo viés holístico, poderá sustentar outras investigações de diferentes naturezas, inclusive, porque, o acesso aberto e a própria comunicação científica são objetos de estudo que atravessam horizontalmente, praticamente, quando não, todas as disciplinas, 
mesmo diante das singularidades de cada uma, ampliando exponencialmente a relevância do presente projeto de pesquisa para o Turismo e outras áreas potenciais; e, de outro, a verificação das "motivações" e "barreiras" dos pesquisadores de Turismo no Brasil em relação à publicação em acesso aberto. De maneira geral, também, a presente pesquisa trará maior visibilidade sobre o tema na área de Turismo (os pesquisadores que não conhecem o movimento poderão integrar o mesmo) e proporcionará dados confiáveis para o futuro planejamento e prognóstico das publicações científicas brasileiras.

Cabe salientar, ainda, que o presente artigo não cobre os aspectos interpretativos dos dados coletados e, também, não adentra as correlações desses com a literatura corrente - por ser uma pesquisa em andamento cujo final está previsto para dezembro de 2016.

\section{Referências}

Anton, B. (2003). Issues and impacts of the changing nature of Scientific Communication. Optometry and Vision Science, 80(6): 403-410.

Atkinson, R.C. (2003). A new world of Scholarly Communication. Chronicle of Higher Education, 50(11): 16.

Bergman, S. (2006). The Scholarly Communication movement: highlights and recent developments. Collection Building, 25(4): 108-128.

Bjork, B.-C. (2007). A Model of Scientific Communication as a global distributed information system. Information Research , 12, jan.

Borgman, C.L. (2000). Digital libraries and the continuum of Scholary Communication. Journal of Documentation, 56(4).

Friedlander, A. (2008). The Triple Helix: Cyberinfrastructure, Scholarly Communication, and trust. Journal of Electronic Publishing, 11.

Fyffe, R. (2002). Technological change and the Scholarly Communications reform movement: reflections on Castells and Giddens. Library Resources and Technical Services, 46(2): 50-61.

Gomes, C.M. (2013). Comunicação Científica: Alicerces, transformações e tendências. Covilhã, UBI, LabCom, Livros LabCom.

Gomes, C.M. (2010). Paradigma do Acesso Aberto (Open Access): alguns apontamentos para os estudiosos do Turismo no Brasil. Turismo em Análise, 21(2). 
Guédon, J.-C. (2001). Oldenburg's long shadow: librarians, research scientists, publishers and the control of scientific publishing. Creating Digital Future, maio.

Hahn, K. (2006). New tools for new times: remodeling the Scholarly Communication system. College \& Research Libraries News, 67(10).

Harnad, S. (2007). Entrevista com Steven Harnad (versão Hélio Kuramoto). Bibli.: R. Eletr. Bibliotecon. Ci. Inf., Florianópolis, n. Esp., $1^{\circ}$ sem.

Harnad, S. (1998). Learned inquiry and the net: the role of peer review, peer commentary and copyright. Learned Publishing, 11(4).

Harnad, S. (1991). Post-Gutenberg galaxy: the fourth revolution in the means of production of knowledge. Public-Access Computer Systems Review, 2(1): 39-53.

Harnad, S. (1990). Scholarly skywriting and the prepublication continuum of scientific inquiry. American Psychological, 1(6).

Harnad, S. (2006). Optimizing OA self-archiving mandates: what? where? when? why? How? Technical Report, ECS, University of Southampton.

Hjorland, B. (2002). Domain analysis in Information Science: eleven approaches

Hurd, J.M. (1996). Models of Scientific Communications systems. S.Y. Crawford; J.M. Hurd \& A.W. Weller, From Print o Electronic: The Transformation of Scientific Communication. USA: Information Today.

Kling, R. (2000). Learning about information technologies and social change: The contribution of social informatics. Information Society, 16(3): 217232.

Kling, R. (2004). The internet and unrefereed scholarly publishing. Annual Review of Information Science and Technology, 38: 591-631, xix-xx.

Lagoze, C. \& Van de Sompel, H. (2001). The Open Archives Initiative: building a low-barrier interoperability framework. Joint Conference on Digital Libraries, 1.

Lynch, C. (2003). The transformation of Scholarly Communication and the role of the libray in the age of networked communication. Serials Librarian, 3: 5-20. 
Meyer, E.T. \& Schroeder, R. (2009). The world wide web of research and access to knowledge. Knowledge Management Research and Practice, 7(3): 218-233.

Odlyzko, A. (2002). The rapid evolution of Scholarly Communication. Learned Publishing, 15: 7-19.

Pikas, C.K. (2006). The impact of information and communication technologies on informal scholarly Scientific Communication: a literature review. Prepared for LBSC878: Doctoral Seminar in Information Studies. USA: University of Maryland College of Information Studies.

Rowlands, I. (2003). Knowledge production, consumption and impact: policy indicators for a changing world. Aslib Proceedings, 55(1-2): 5-12.

Schroeder, R. \& Fry, J. (2007). Social science approaches to e-science: framing an agenda. Journal of Computer-Mediated Communication, 12(2).

Sondergaard, T.; Andersen, J. \& Hjorland, B. (2003). Documents and the communication of scientific and scholarly information revising and updating the UNISIST Model. Journal of Documentation, 59: 278-320.

Tenopir, C. \& King, D.W. (2000). Towards electronic journals - realities for scientists, librarians and publishers. USA: Special Libraries Association.

Vickery, B.C. (2000). Scientific Communication in history. London: The Scarecrow Press. 